\title{
Rapid carbon nanotubes suspension in organic solvents using organosilicon polymers
}

Federico Dalcanale ${ }^{\mathrm{a}, \mathrm{b},{ }^{*}}$, Jonas Grossenbacher ${ }^{\mathrm{c}}$, Gurdial Blugan ${ }^{\mathrm{a}}$ Maurizio R. Gullo ${ }^{\mathrm{c}}$, Jürgen

Brugger $^{\mathrm{c}}$, Hendrik Tevaearai ${ }^{\mathrm{d}}$, Thomas Graule ${ }^{\mathrm{a}}$, Jakob Kuebler ${ }^{\mathrm{a}}$

${ }^{a}$ Empa, Laboratory for High Performance Ceramics, Swiss Federal Laboratories for Materials Science and Technology, Ueberlandstrasse 129, Duebendorf, Switzerland

${ }^{b}$ Laboratory for Multifunctional Materials, Department of Materials, ETH Zürich, Vladimir-Prelog-Weg 5, 8093 Zürich, Switzerland

${ }^{c}$ Microsystems Laboratory, EPFL, Station 17, CH-1015 Lausanne, Switzerland

${ }^{\mathrm{d}}$ Clinic for Cardiovascular Surgery, Inselspital Berne, Berne University Hospital and University of Berne, Berne, Switzerland

Authors E-mail address: dalca82@hotmail.com (Federico Dalcanale, " ionas.grossenbacher@epfl.ch (Grossenbacher Jonas);

Gurdial.Blugan@empa.ch (Blugan, Gurdial);

rosario.gullo@rudevoltage.ch (Maurizio R. Gullo);

juergen.brugger@epfl.ch (Brugger Jürgen);

Hendrik.TevaearaiStahel@insel.ch (Tevaearai Hendrik);

Thomas.Graule@empa.ch (Graule Thomas);

Jakob.Kuebler@empa.ch (Kübler Jakob) 


\begin{abstract}
A strategy for a simple dispersion of commercial multi-walled carbon nanotubes (MWCNTs) using two organosilicones, polycarbosilane SMP10 and polysilazane Ceraset PSZ20, in organic solvents such as cyclohexane, tetrahydrofurane (THF), m-xylene and chloroform is presented. In just a few minutes the combined action of sonication and the presence of $\operatorname{Pt}(0)$ catalyst is sufficient to obtain a homogeneous suspension, thanks to the rapid hydrosilylation reaction between $\mathrm{Si}-\mathrm{H}$ groups of the polymer and the CNT sidewall. The as-produced suspensions have a particle size distribution $<1 \mu \mathrm{m}$ and remain unchanged after several months. A maximum of 0.47 and $0.50 \mathrm{mg} / \mathrm{ml}$ was achieved, respectively, for Ceraset in THF and SMP10 in chloroform. Possible applications as polymeric and ceramic thin films or aerogels are presented.
\end{abstract}

Keywords: MWCNT suspension, hydrosilylation, polycarbosilane, polysilazane, aerogel 


\section{Introduction}

Accomplishment of a homogeneous filler dispersion is the key to produce a high performing composite. The mechanical strength is improved and percolation is achieved at lower volumes. This is true in general but it assumes more significance in the case of carbon nanotubes (CNTs). With exceptional properties such as Young's modulus in the order of $1 \mathrm{TPa}$, electrical conductivity between $10^{2}-10^{6} \mathrm{~S} / \mathrm{cm}$ combined with a high aspect ratio, makes CNTs attractive for a broad range of applications. Their agglomeration-free dispersion in the matrix is important for achieving the maximum effect. Well dispersed systems can reach percolation already at 0.025 wt. \% as reported by Sandler et al. for a CNT-epoxy composite[1]. On the contrary agglomerates of CNTs act as defects causing stress concentration and premature failure of the structure under mechanical load.

A common strategy for producing polymer composites is solution blending. Briefly, the polymer is added while stirring in a CNTs suspension followed by solvent evaporation. Functionalized CNTs or surfactants are used to improve the dispersion. Also in-situpolymerization is based on the same strategy but in this case there is formation of chemical bonds between the monomer and functionalized CNT surface as reported for epoxy nanocomposites[2]. To preserve the electronic structure of the CNTs noncovalent dispersing methods were investigated to avoid the functionalization step that is reported to damage it. Another reason is the simplification of the whole process. Functionalization of CNTs involves the use of acids plus a washing/drying procedure before their effective use[3]. As reported by Bilalis et al. [4] polymers can directly dissolve untreated CNTs in solvents via noncovalent $\pi$ $\pi$ and $\mathrm{CH}-\pi$ interactions $[5,6]$ but also wrapping[7]. The polymer is adsorbed on the surface of the CNTs, avoiding the re-aggregation process in liquid media due to the steric repulsion exerted. The use of conventional un-modified polymers and to directly disperse CNTs in organic solvents via $\mathrm{CH}-\pi$ interactions seems the most interesting approach because no particular procedures have to be adopted. However, the dispersibility reported[6] is very low 
(below $0.020 \mathrm{mg} / \mathrm{ml}$ ) for practical use in a composite. A method to improve it up to $1.235 \mathrm{mg} / \mathrm{ml}$ was found by Xu et al. in 2009 [8] using a hyperbranched polyethylene. The branched structure was suggested to cause a higher $\mathrm{CH}-\pi$ interaction enhancing the solubility. Interesting properties due to the polymer architecture were also found with dendrimers [9].

Despite all these different approaches and possibilities there are still few works about the use of organosilanes for CNTs suspension and among them almost only polydimethylsiloxane (PDMS) is investigated. To the best of our knowledge there are no reports about the use of other organosilanes as dispersants for CNTs. Among them preceramic polymers polysilazane, polyborosilane and polycarbosilane are an important class because technical ceramics such as $\mathrm{SiC}, \mathrm{SiCN}, \mathrm{SiCBN}$ can be obtained after pyrolysis. These are also promising materials for diverse applications such as micro-electro-mechanical-system (MEMS) fabrication[10, 11], anode batteries[12], harsh environment sensors[13] and high temperature resistant fibers[14]. The first study about polysilazane-CNT composites[15] was in 2004 and up to now only few others followed[16-21]. The filler dispersion in the polymer matrix is usually poorly described and realized following different methods such as functionalized CNTs, surfactants or direct sonication of the filler in organic solvents. All these techniques involve either a long procedure or are not efficient.

In this paper we report a simple one-step method for the dispersion of pre-sonicated CNTs in organic solvents. We show for the first time that preceramic polymers and in particular polycarbosilane SMP10 can be used to disperse MWCNTs thanks to the addition of $\mathrm{Pt}(0)$ as catalyst during sonication. The main advantage compared to other methods is the simplicity of the process. All the used products are commercially available and no additional steps such as washing, drying and functionalization are needed except solvent removal to obtain a CNT/polymer mixture ready for casting. The polymer used is in fact also the matrix of the composite and $\operatorname{Pt}(0)$ the catalyst to aid curing. In this way we produced a polymer-MWCNT 
stable suspension that can be used in various applications. The concept was then also shown to be valid for the polysilazane Ceraset.

\section{Experimental procedure}

\subsection{Materials used}

Two different types of commercially available MWCNT were used as received. Baytubes C150P (Bayer Material-Science AG, Leverkusen, Germany) that possess purity > 95 wt. \% and tube length $>1 \mu \mathrm{m}$. Nanocyl ${ }^{\mathrm{TM}}$ NC7000 (Nanocyl S.A., Sambreville, Belgium) that possess purity $>90$ wt. $\%$ and average tube length of $1.5 \mu \mathrm{m}$. Both are produced via catalytic chemical vapor deposition (CVD) methods. More information can be found in the respective datasheets. The two commercially available preceramic polymers (PDC polymers) used were allylhydridopolycarbosilane SMP10 (batch J20867, viscosity 51 cps Starfire Systems Inc., USA) with nominal formula $\left[\mathrm{SiH}\left(\mathrm{CH}_{2} \mathrm{CH}=\mathrm{CH}_{2}\right) \mathrm{CH}_{2}\right]_{0.1}\left[\mathrm{SiH}_{2} \mathrm{CH}_{2}\right]_{0.9}$ and polysilazane Ceraset PSZ20 (Kion Corp. Pennsylvania, USA) with nominal formula $\left[\mathrm{SiCH}_{3}\left(\mathrm{CH}_{2} \mathrm{CH}=\mathrm{CH}_{2}\right) \mathrm{NH}\right]_{0.2}\left[\mathrm{SiCH}_{4} \mathrm{NH}\right]_{0.8}$.

Platinum(0)-1,3-divinyl-1,1,3,3-tetramethyldisiloxane complex solution $2 \%$ in xylene (Sigma Aldrich, Switzerland), further diluted in m-xylene to obtain a concentration of $0.1 \mathrm{wt} . \%$ and used as catalyst.

All the solvents used (cyclohexane, m-xylene, THF, chloroform) had a purity $>99.5 \%$ and were purchased in Switzerland from Sigma Aldrich or Carl Roth.

\subsection{MWCNT de-bundling}

The MWCNTs were first separated into individual nanotubes de-bundling the as purchased powder via sonication in the selected organic solvent for $2 \mathrm{~h}$ using a Sonoplus 2200 (Bandelin, Germany) set with cycle 9 , power $20 \%$ and equipped with a titanium probe. The process was performed in a water bath cooled with ice to avoid excessive evaporation of the solvent. The 
solvent weight loss was compensated by adding fresh solvent to obtain a final CNT concentration of $3.9 \mathrm{mg} / \mathrm{ml}$. Typically, a batch of about $10.5 \mathrm{ml}$ of de-bundled CNT in solvent was produced each time.

\subsection{Suspension preparation}

The preceramic polymer was first dissolved in the organic solvent and de-bundled MWCNT added, reaching the desired CNT concentration. Finally $\operatorname{Pt}(0)$ catalyst was added. The mixture was first homogenized by hand and then sonicated with a VC70C ultrasonic processor (Sonics, USA) set at pulse 6 and amplitude $40 \%$ and equipped with a Vibracell VC18 probe (Sonics, USA). The suspension was stored refrigerated for at least $12 \mathrm{~h}$ to allow formation of aggregates and then centrifuged with a Universal 320 (Hettich, DE) for $30 \mathrm{~min}$ at $4000 \mathrm{rpm}$ (2701 $\mathrm{RCF}_{\mathrm{MAX}}$ ). The extracted supernatant was then analyzed.

In a typical preparation, a $0.50 \mathrm{mg} / \mathrm{ml} \mathrm{CNT} \mathrm{suspension} \mathrm{in} \mathrm{cyclohexane} \mathrm{was} \mathrm{obtained} \mathrm{sonicating}$ $150 \mu 1$ of SMP10 added together with $3290 \mu$ of pure cyclohexane, $514 \mu$ l of de-bundled CNT in cyclohexane and $50 \mu \mathrm{l}$ of $\operatorname{Pt}(0) 0.1$ wt. \% in m-xylene. A scheme of the whole suspension preparation is presented in Figure 1.

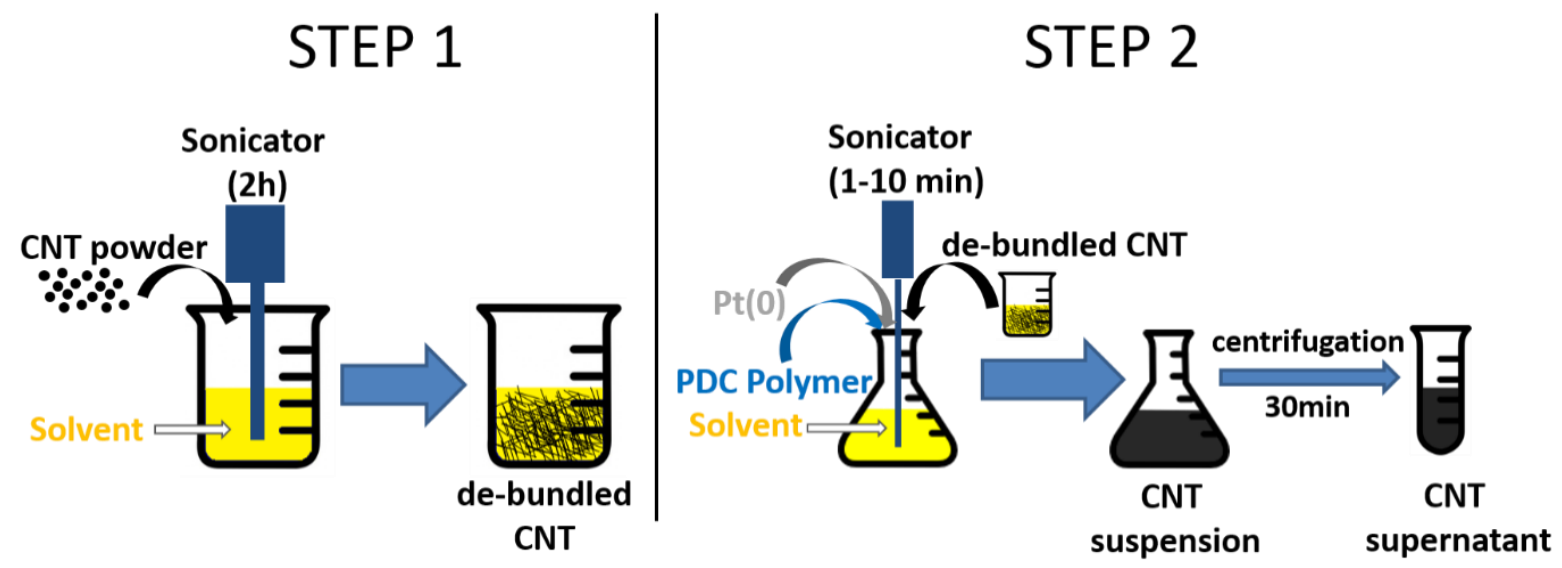

Figure 1: Scheme of the suspension process: de-bundling of CNT powder STEP 1). Suspension realization STEP 2) At the end of step 2 the suspension is centrifuged and the supernatant extracted 


\subsection{Characterization methods}

- Fourier transform infrared spectroscopy (FT-IR) spectra were collected on a Tensor 27 (Bruker, MA, USA) pouring some sample drops on a Golden Gate ATR.

- Intensity weighted particle size distribution was analyzed with a Zetasizer Nano S (Malvern, UK) by dynamic light scattering (DLS) in NIBS (non invasive back scattering) configuration and in accordance with ISO 22412 as previously described[22]. For this measurement the suspension was diluted to obtain a semi-transparent liquid. The distribution data are an average of a minimum of three different runs each one composed by 13 autocorrelation functions (ACFs) combined to a mean ACF and evaluated by the Zetasizer Software version 7.03 appendant to the Zetasizer Nano S.

- Particle size distribution of the de-bundled CNT was measured with light scattering LS320 (Beckman Coulter, USA).

- Uv-Vis analysis were performed in a quartz glass cuvette with a Uv-3600 (Shimadzu, Japan) operating between $200-850 \mathrm{~nm}$. Absorbance was maintained between 0.1-2 in the whole range used for the calculations through proper dilution as described previously[23]. Solvent and cuvette contribution were subtracted from the resulting spectrum.

- Thermal gravimetric analysis was conducted under flowing air using a TGA/sDTA 851e (Mettler Toledo, OH, USA) and applying a $5^{\circ} \mathrm{C} / \mathrm{min}$ heating rate up to $1000^{\circ} \mathrm{C}$. The suspended CNTs were analyzed after being washed from the polymer excess. This procedure can be described as follow: the supernatant was centrifuged at $15000 \mathrm{rpm}\left(21382 \mathrm{RCF}_{\mathrm{MAX}}\right)$ for at least 30-60 min until complete CNT settling. The solvent was then replaced with fresh one and the settled CNT re-dispersed for 1 minute with a Vibracell VC 18 (Sonics, USA) sonication finger. This procedure was repeated three times. At the end no significative intensity differences indicating the presence of residual SMP10 between fresh and centrifuged solvent were detected with FT-IR/ATR. 
${ }^{1} \mathrm{H}$ NMR spectra were recorded at $400.18 \mathrm{MHz}$ on a Bruker Avance III $400 \mathrm{NMR}$ spectrometer. The $1 \mathrm{D}{ }^{1} \mathrm{H}$ NMR spectra were performed at $298 \mathrm{~K}$ using a $5 \mathrm{~mm}$ BBO Prodigy ${ }^{\mathrm{TM}}$ CryoProbe with z-gradient and $90^{\circ}$ pulse lengths of $11.4 \mu \mathrm{s}\left({ }^{1} \mathrm{H}\right), 10.0 \mu \mathrm{s}\left({ }^{13} \mathrm{C}\right)$ and $13.0 \mu \mathrm{s}\left({ }^{29} \mathrm{C}\right)$. All spectra were recorded with Bruker standard pulse programs and parameter sets, and the ${ }^{1} \mathrm{H}$ chemical shifts were referenced internally using the resonance signals of cyclohexane-d $\mathrm{d}_{12}$ at $1.38 \mathrm{ppm}$.

\section{Results}

\subsection{MWCNT-SMP10 suspension in cyclohexane}

Among the organic solvents available, cyclohexane was analyzed first for several reasons. It is a common organic solvent, both catalyst and polymer are completely soluble, and it is not miscible with water, limiting undesired hydrolysis and condensation reactions. It has a relatively low boiling point $\left(80.7^{\circ} \mathrm{C}\right)$ that simplifies its extraction and a high melting point $\left(6.5^{\circ} \mathrm{C}\right)$ that allows it to be easily stored avoiding evaporation. In addition it has low toxicity and it is cheap.

On the other hand, as is visible in Figure 2D, CNTs have negligible solubility in it and settle in less than $1 \mathrm{~h}$ after sonication is stopped. A prolonged sonication is also ineffective to stabilize them. Similarly, when SMP10 is added to the solvent in Figure 2E, CNT settling occurs as well. The result completely changes in Figure $2 \mathrm{~F}$ when $\mathrm{Pt}(0)$ is added to cyclohexane together with SMP10 and a dark suspension is obtained after a short sonication time. As shown in Figure 2F the suspension is still dark after one month and has been observed to remain unchanged, without visible phase separation, for several months until the complete evaporation of the solvent. Complete CNT settling could be archived only after centrifuging it at $15^{\circ} 000 \mathrm{rpm}(21,382$ $\left.\mathrm{RCF}_{\mathrm{MAX}}\right)$ for at least $30 \mathrm{~min}$. 


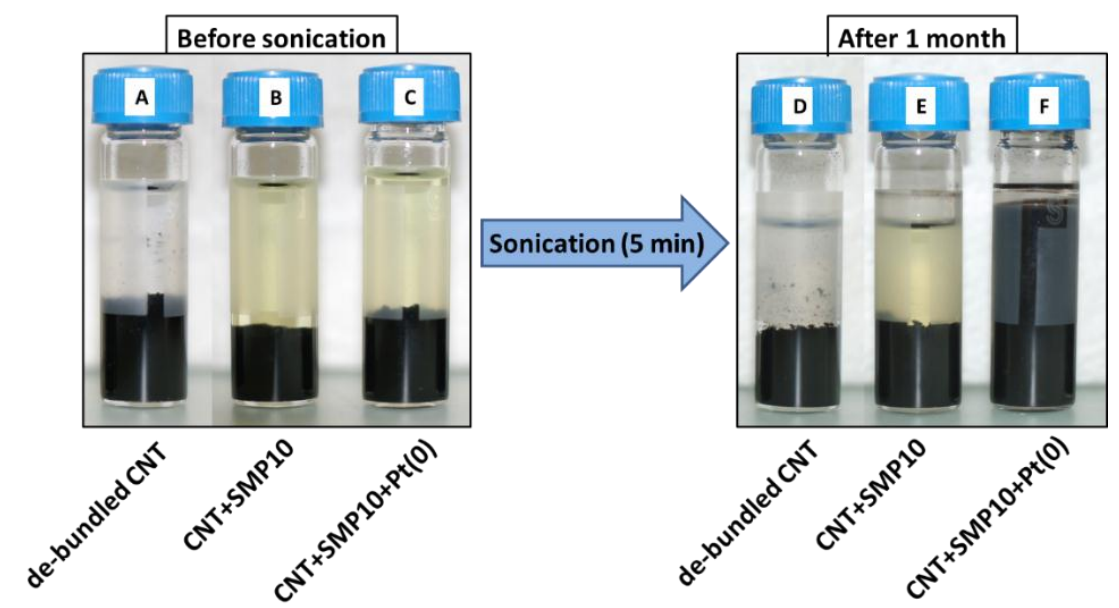

Figure 2: CNT in cyclohexane. Effect of SMP10 and catalyst Pt(0) addition to promptly suspend MWCNT after few minutes of sonication. The difference in solvent level is due to evaporation during storage.

The difference in particle size distribution of the CNT before and after the suspension step is shown in Figure 3. Initially the distribution in the de-bundled CNT is broad, with $\mathrm{d}_{10}=2.7 \mu \mathrm{m}$ and $\mathrm{d}_{90}=26.6 \mu \mathrm{m}$. The aggregates due to Van der Waals' interactions have a mean size of about $14,5 \mu \mathrm{m}$ in terms of hydrodynamic diameter $\mathrm{D}_{\mathrm{h}}$. After the addition of $\operatorname{SMP} 10, \operatorname{Pt}(0)$ and sonication for a few minutes the whole distribution, measured with Dynamic Light Scattering, is shifted below $1 \mu \mathrm{m}$ and the mean size is about $250 \mathrm{~nm}$. The distribution is comparable with the one obtained by Krause et al.[22] for Baytubes dispersed with sodium dodecyl benzene sulfonate (SDDBS) and it was associated solely to the presence of single-CNTs in the solution.

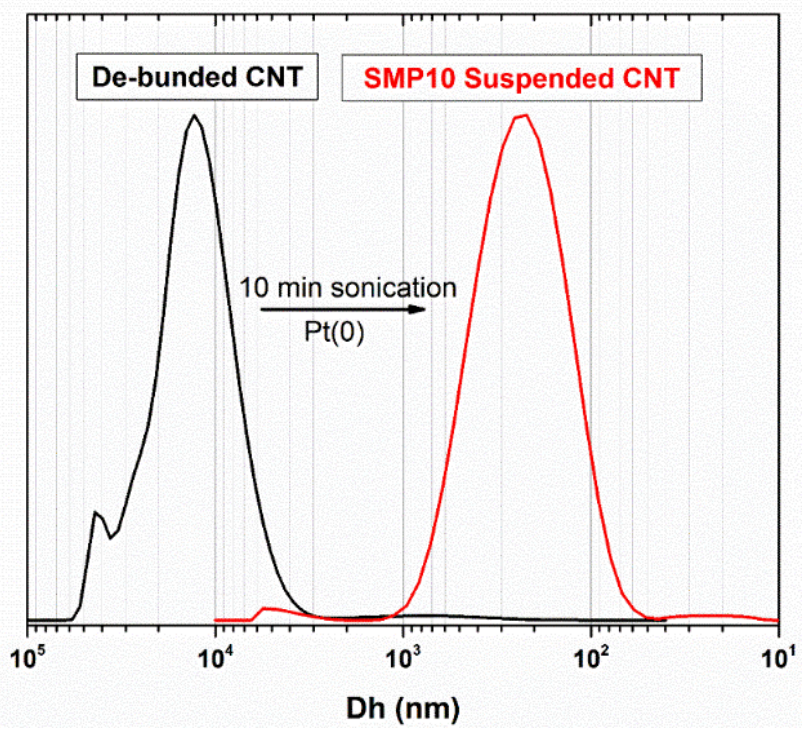

Figure 3: particle size distribution of CNT before and after sonication in the presence of SMP10 and $\mathrm{Pt}(0)$. 


\subsection{Analysis of the suspension parameters}

Dispersion of CNTs can be characterized using UV-vis absorption spectroscopy. Absorption values at $600 \mathrm{~nm}$ were recorded and directly related to the amount of single CNTs in solution according to the Lambert-Beer $\operatorname{law} \mathrm{A}=\log \mathrm{I} / \mathrm{I}=\boldsymbol{\varepsilon} \cdot \mathrm{C} \cdot 1$ where $\mathrm{A}$ is the measured absorbance and $\mathrm{C}$ the unknown concentration. The light path length 1 is fixed by the experimental setup while the calculated extinction coefficient for MWCNT at $600 \mathrm{~nm}$ was $\varepsilon_{600}=0.0385 \mathrm{~L} /(\mathrm{mg} \cdot \mathrm{cm})$. At this wavelength polymer and solvents are not active and the only contribution to the absorption is attributed to the presence of individual CNTs. Bundles are in fact reported to not be active in the UV-vis region [24]. The efficacy of the dispersion method was evaluated defining its extractability as previously applied to surfactant-CNT suspensions [25] and expressed as:

$$
\% \text { extractability }=\frac{C}{C_{0}} \times 100
$$

where $\mathrm{C}$ is the concentration of suspended MWNTs measured with UV-vis and $\mathrm{C}_{0}$ the concentration of MWNTs originally in solvent. This parameter is used to determine the effect of the process variables and to find the optimum conditions. The extractability values were taken as an average of at least three samples each produced from a different batch of de-bundled CNTs. In Figure 4 the influence of catalyst amount is analyzed. The solid and dashed lines compare, respectively, the extractability of the suspension produced with and without the use of $\mathrm{Pt}(0)$. The initial CNT concentration was $0.50 \mathrm{mg} / \mathrm{ml}$. All the other variables such as solvent amount, polymer content, sonication time and power are kept constant. As it is visible, without catalyst the extractability of the system ranges between 1-7\%. Aggregates are easily formed and precipitate during the centrifugation step, leaving an almost clear supernatant. On the contrary, the addition of catalyst to the system promptly improves the extractability up to $65 \%$ at $116 \mathrm{ppm}$. For higher $\mathrm{Pt}(0)$ amounts, up to $638 \mathrm{ppm}$, the solid curve stabilizes in a range between $60-65 \%$. The considerable large standard deviation displayed is mainly attributed to the different amount and size of well separated CNT obtained during the de-bundling step that 
was not fully optimized in this work. As reported for surfactant based dispersions[26-28], changes in the process parameters such as probe position, power and temperature affect the degree of de-bundling. In the best case the maximum extractability on a single batch was calculated up to about $82 \%$, that corresponds to a CNT concentration of $0.41 \mathrm{mg} / \mathrm{ml}$. The extent of the standard deviation seems to increase also accordingly with the catalyst amount and it is explained by the higher number of reactions taking place and the increasing complexity of the system.

To determine the suspension mechanism, the effect of platinum addition was studied with TGA and it is shown in Figure 4B. The analyzed CNTs were heated under air stream up to $900{ }^{\circ} \mathrm{C}$ with a rate of $5{ }^{\circ} \mathrm{C} / \mathrm{min}$. As purchased Baytubes were also analyzed for comparison. Pure carbon nanotubes decompose in air between $400{ }^{\circ} \mathrm{C}$ and $600{ }^{\circ} \mathrm{C}$ leaving a residue mass of $3 \%$ that is compatible with the $>95 \%$ purity indicated in the datasheet and measured with the same method. When only SMP10 is added, MWCNTs decompose at a higher temperature between $450{ }^{\circ} \mathrm{C}-600{ }^{\circ} \mathrm{C}$ with a residue mass of $36.5 \%$. This is attributed to the presence of polycarbosilane that undergoes an oxidation process forming infusible $\mathrm{Si}-\mathrm{C}_{\mathrm{x}}-\mathrm{O}_{\mathrm{y}}$ as described previously[29, 30]. With the addition of $290 \mathrm{ppm}$ of $\mathrm{Pt}(0)$, red line, the residues mass increases to $49 \%$ with a shift of the oxidation process at even higher temperatures between 510 and 640 ${ }^{\circ} \mathrm{C}$.
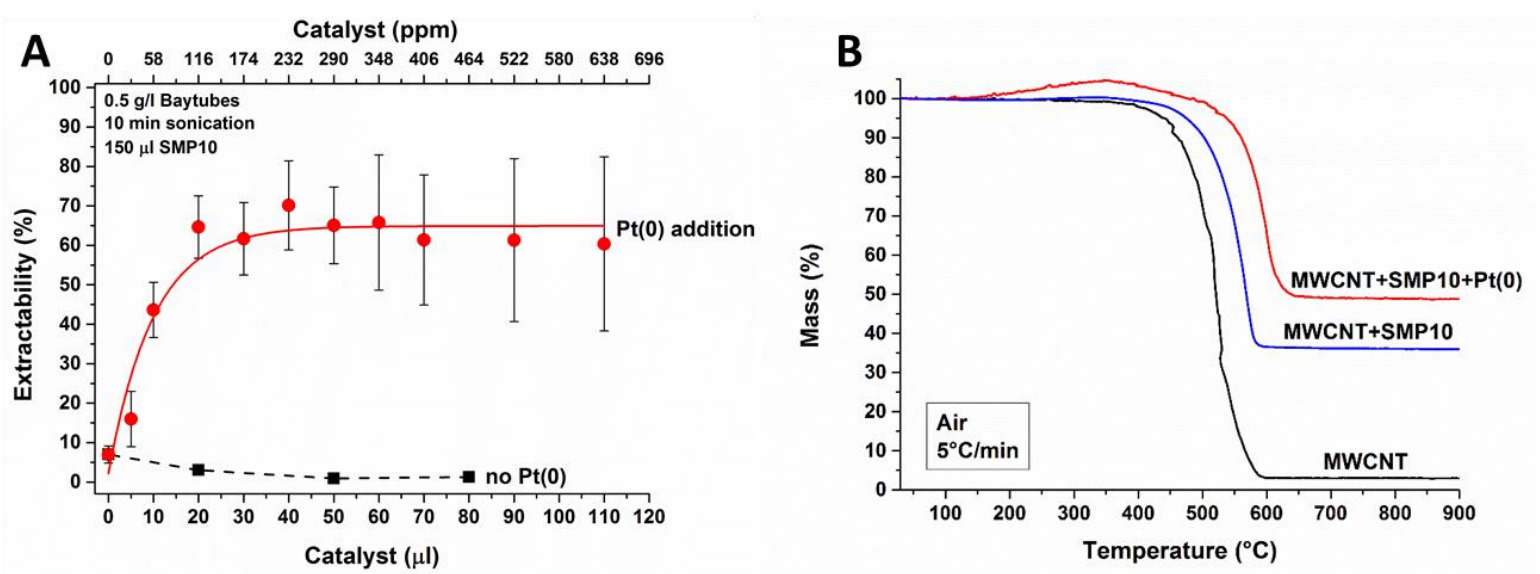

Figure 4: A) Effect of $\mathrm{Pt}(0)$ addition on the extractability of a CNT suspension in cyclohexane. B) TGA of MWCNT+SMP10 with and without addition of $290 \mathrm{ppm}$ of $\mathrm{Pt}(0)$ after removal of polymer excess. Pristine Baytubes mass loss is also shown. 
The catalyst amount in the suspension was then fixed at $290 \mathrm{ppm}$ to maintain a high extractability and analyze the effect of the ultrasonication time. As is visible in Figure 5A, after 1 minute the extractability is already $28 \%$ and it increases up to $65 \%$ after 10 min when the separation of CNT can be considered almost completed. Further sonication in fact improves this value to $74 \%$ but with a considerable reduced rate. To determine the optimum nominal $\mathrm{CNT} /$ polymer ratio and maximize the amount of suspended CNT compared to the polymer used, the initial CNT concentration was varied between 0.25 and $1 \mathrm{mg} / \mathrm{ml}$ whilst the SMP10 amount of $150 \mu \mathrm{l}$ was kept constant. As visible in the blue line in Figure 5B the optimum was calculated with a Gaussian fit to be about 1.68 wt. \%, that corresponds to an initial CNT amount of $0.67 \mathrm{mg} / \mathrm{ml}$. This gives at the end of the process a supernatant CNT concentration of about $0.33 \mathrm{mg} / \mathrm{ml}$ that correspond to an extractability of $60 \%$. In the same Figure 5B the extractability displayed as a black dashed curve decreases progressively with the CNT amount from a maximum of $73 \%$ to $25 \%$.

The same suspension process was also tested using commercial Nanocyl 7000 powder as MWCNT source. Starting from a concentration of $0.50 \mathrm{mg} / \mathrm{ml} \mathrm{CNT} \mathrm{in} \mathrm{cyclohexane} \mathrm{in} \mathrm{presence}$ of $150 \mu 1 \mathrm{SMP} 10$ the effect of $\operatorname{Pt}(0)$ addition was tested. In the same way as Baytubes, the catalyst was fundamental to achieve a stable suspension that otherwise couldn't be obtained. Extractability was calculated to be $51 \%$ after 10 min sonication and mean particle size $335 \mathrm{~nm}$ with the whole distribution lower than $1 \mu \mathrm{m}$.
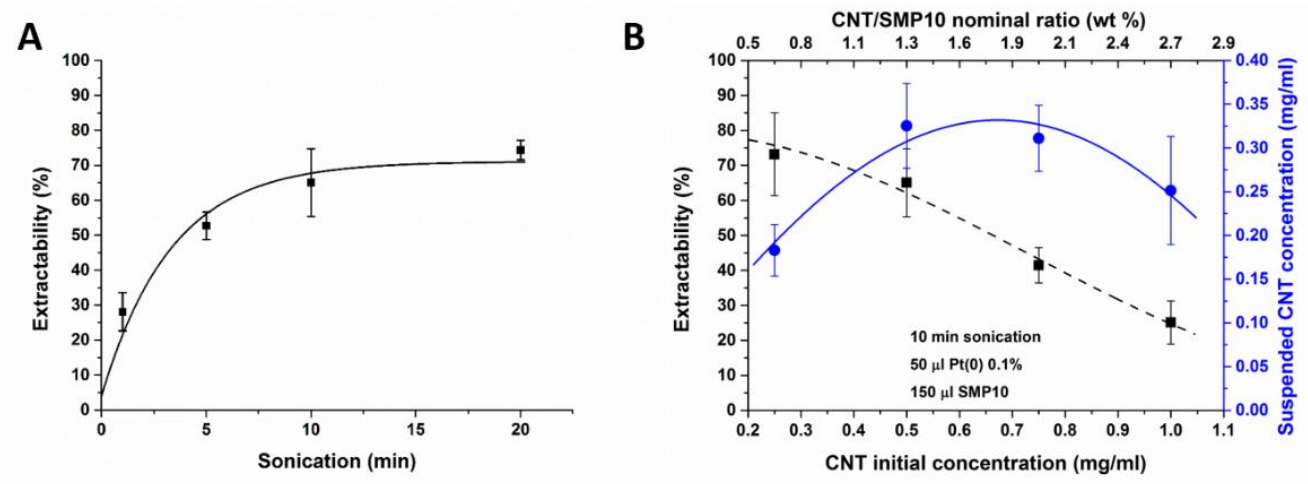

Figure 5: Analysis of process parameters on extractability in cyclohexane. A) Effect of sonication time B) effect of initial CNT concentration. 


\subsection{SMP10-MWCNT suspension in m-Xylene, THF, Chloroform}

Starting from a CNT concentration of $0.50 \mathrm{mg} / \mathrm{ml}$ and maintaining the same process parameters (150 $\mu 1$ of SMP10, $290 \mathrm{ppm} \operatorname{Pt}(0), 10$ min sonication), the effect of organic solvents other than cyclohexane on the suspension was analyzed. As shown in Figure 6A and B sedimentation occurs in THF and m-xylene a few hours after sonication and it's independent of $\operatorname{Pt}(0)$ use. On the contrary in Figure 6C a black stable solution with extractability of $90-100 \%$ and mean particle size of $350 \mathrm{~nm}$ could be obtained in chloroform. As shown in Figure 6D a stable suspension is obtained in chloroform also without addition of $\operatorname{Pt}(0)$. In this case, despite the absence of a clear sedimentation process aggregates together with single CNT were noticed after proper dilution.

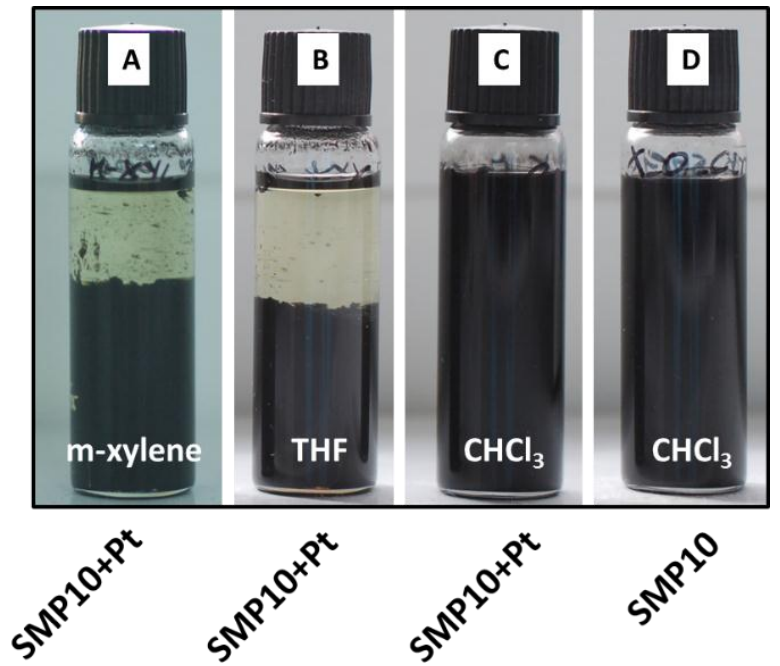

Figure 6: SMP10-MWCNT suspensions in m-xylene, THF and chloroform $24 \mathrm{~h}$ after sonication. CNT initial concentration is $0.50 \mathrm{mg} / \mathrm{ml}$

\subsection{Ceraset-MWCNT suspensions in organic solvents.}

In liquid form like SMP10, Ceraset PSZ20 is soluble in the same solvents and its polymer chain structure includes $\mathrm{Si}-\mathrm{H}, \mathrm{C}-\mathrm{H}$ and vinyl groups together with $\mathrm{N}-\mathrm{H}$ group characteristic of polysilazanes. The efficacy of the suspension method schematized in Figure 1 with and without the addition of $290 \mathrm{ppm} \operatorname{Pt}(0)$ was tested in several organic solvents and the results are summarized in Table 1. Extractability and mean particle size are reported as well as the type of CNT used. 
In cyclohexane and chloroform a stable CNT suspension with a particle size distribution $<1 \mu \mathrm{m}$ was obtained in the presence of $\operatorname{Pt}(0)$. No aggregates were formed and the extractability was $57 \%$ and $51 \%$ respectively. Without catalyst large aggregates were instead found even after centrifuging at $4000 \mathrm{rpm}$ for $30 \mathrm{~min}$, the suspension remained black. Similar results were obtained with Nanocyl, where mean particle size and extractability in cyclohexane were 461 $\mathrm{nm}$ and $49 \%$, respectively.

In THF stable CNT suspension with mean particle size of $298 \mathrm{~nm}$ was obtained without addition of $\operatorname{Pt}(0)$. The extractability was measured to be $57 \%$ and no aggregates were noticed after dilution. With the addition of catalyst, the particle distribution remains similar with a mean size of $254 \mathrm{~nm}$, but the extractability increases up to $95 \%$.

An opposite case is represented by m-xylene, where despite the use of $\operatorname{Pt}(0)$ complete settling and aggregation takes place soon after the sonication stops.

\begin{tabular}{|ccccc|}
\hline Solvent & $\begin{array}{c}\text { Catalyst } \\
\mathbf{2 9 0} \mathbf{~ p p m}\end{array}$ & $\begin{array}{c}\mathbf{C N T} \\
\mathbf{0 , 5} \mathbf{~ m g} / \mathbf{m l}\end{array}$ & Extractability & Mean Size \\
\hline Cyclohexane & YES & Baytubes & $57 \%$ & $381 \mathrm{~nm}$ \\
\hline Cyclohexane & YES & Nanocyl & $49 \%$ & $461 \mathrm{~nm}$ \\
\hline Cyclohexane & NO & Baytubes & - & Aggregates \\
\hline Chloroform & YES & Baytubes & $51 \%$ & $245 \mathrm{~nm}$ \\
\hline Chloroform & NO & Baytubes & - & Aggregates \\
\hline THF & YES & Baytubes & $95 \%$ & $254 \mathrm{~nm}$ \\
\hline THF & NO & Baytubes & $57 \%$ & $298 \mathrm{~nm}$ \\
\hline m-xylene & YES & Baytubes & - & Aggregates \\
\hline m-xylene & NO & Baytubes & - & Aggregates \\
\hline
\end{tabular}

Table 1: extractability and mean particle size of CNT suspensions in several organic solvents using Ceraset PSZ20. 


\section{Discussion}

\subsection{MWCNT-SMP10 suspension in cyclohexane}

CNT suspension using organosilicones raises several questions about the role of the catalyst and the solvents in the process. As shown in Figure 2D a stable CNT suspension can not be achieved in pure organic solvents such as cyclohexane even after intensive sonication. The high surface area leads the nanotubes to form agglomerates via Van der Waals forces and then precipitate over time. Common strategies to keep single nanotubes in solution are steric and electrostatic repulsion[31]. This is commonly used for nanoparticles in water, adjusting the $\mathrm{pH}$ to maximize the Zeta potential but cannot be used in organic solvents. The only option is to use a polymer that covers the nanoparticles avoiding their direct interaction.

From the results obtained with SMP10 we can assume that nanotube coverage is not caused by a single mechanism such as $\pi-\pi$ interaction, covalent bonding or polymer structure but is the combination of several mechanisms working together. Starting from the point that a suspension process is a competition between the interaction of polymer-CNT and polymer-solvent, when the latter is stronger than the former, reduced or inefficient solubilization occurs $[7,41]$. This is the case of suspensions without $\mathrm{Pt}(0)$ addition. The $36.5 \%$ TGA yield in Figure 4B obtained after the removal of excess polymer denotes that part of it adheres onto the CNT surface. This is possible if we assume the existence of a non-covalent interaction between SMP10 and CNTs. This is probably due to the abundant $\mathrm{CH}$ bonds in the polycarbosilane chain structure that give place to $\mathrm{CH}-\pi[6,8,32]$ interactions with the CNT sidewall. As reported by Beigbeder et al. [33] $\mathrm{CH}-\pi$ are responsible for the absorption properties of PDMS on CNT and are described as weak hydrogen bonds between soft acids and soft bases, largely due to dispersion forces and partly to charge-transfer and electrostatic forces. This interaction is probably also enhanced by the hyperbranched structure of the polycarbosilane[34] as reported for similar polymers[8, 35]. 
SMP10 alone is insufficient to efficiently cover the nanotube surface and this causes precipitation as shown in Figure 2E. To obtain a stable suspension a "critical surface coverage" defined as the minimum polymer amount necessary on the CNT surface to avoid CNT aggregation, must be reached. This condition is met with the addition of $\operatorname{Pt}(0)$ where a higher CNT coverage, displayed by a $12.5 \%$ higher yield in Figure 4B, can be achieved. This is also visible with the stabilization of the nanotubes in Figure 2E and the drastic change in particle size distribution in Figure 3. The effect of $\mathrm{Pt}(0)$ is most likely to promote a hydrosilylation reaction by lowering the activation energy between the SMP10 Si-H groups and the CNT double bonds, thus allowing a higher and more stable coverage of the nanotubes. $\operatorname{Pt}(0)$ is a wellknown Karstedt's catalyst specific for hydrosilylation[36], used in the curing of silicones and this reaction has already been reported to occur spontaneously at room temperature between triethylsilane and metallic SWCNT[37]. Due to the wrapping of the polymer on the nanotube, the reaction is supposed to involve only a small fraction of the $\mathrm{Si}-\mathrm{H}$ groups available, those closest to the CNT wall. This would explain the absence of significative changes of the Si-H signal intensity in the ${ }^{1} \mathrm{H}$ NMR spectra (attached in the supplementary information) between the diluted polymer and the same, sonicated in the presence of $300 \mathrm{ppm}$ of $\mathrm{Pt}(0)$ and CNT.

Starting from the TGA data the polymer amount on the nanotubes can be estimated in terms of monolayers (ML $\left.\mathrm{MiC}_{\mathrm{S}}\right)$ using the formula reported by Shah et al.[38].

$$
M L_{S i C}=\frac{\omega_{S i C}}{1-\omega_{S i C}} \times \frac{M W_{C}}{M W_{S i C}} \times\left(\frac{\Omega_{S i C}}{\Omega_{C}}\right)^{2 / 3}
$$

Where $\omega_{\mathrm{SiC}}$ is the ceramic yield at $900^{\circ} \mathrm{C}$ without contribution of the $\mathrm{CNT}$ impurities, MW and $\Omega$ are molecular weight and molar volume/Avogadro's number respectively. The subscripts denotes the carbon in the nanotubes and the ceramic molecules of the TGA residues. It has to be mentioned that due to the impossibility to examine the small TGA residues and the difference that may exist between monolayer and bulk specimens pyrolysis, the above formula gives only an approximation of the polymer coverage. Due to the multitude of literature data, given by 
different process conditions and precursor chemistry used, we assumed for the ceramic a density between 2.4-2.8 $\mathrm{g} / \mathrm{cm}^{3}$ [39-41] and a composition ranging between $\mathrm{SiC}_{1.19} \mathrm{O}_{0.16}$ and $\mathrm{SiC}_{1.16} \mathrm{O}_{0.76}$ as reported previously for the same[42] and other precursors [43-46], within which our composition falls. The density of carbon is taken as $2.26 \mathrm{~g} \cdot \mathrm{cm}^{-3}$ and its atomic volume as 0.0088 $\mathrm{nm}^{3}$. With these values $\mathrm{ML}_{\mathrm{SiC}}$ is calculated to be $0.5-0.55$ and $0.25-0.3$, respectively with and without $\operatorname{Pt}(0)$ addition. This shows that there is a non-covalent interaction between polymernanotubes, confirming what was reported for $\mathrm{SiCN}$ precursor[38], and that $\operatorname{Pt}(0)$ addition can double the polymer coverage on the nanotubes allowing their dispersion. The calculated values are most likely higher because the fraction of the non-covalent coverage may have been removed during the washing procedure.

The catalyst is then the key factor to stabilize the nanotubes and its amount influences the process and the extractability. In Figure 4A after 10 min sonication the system reaches its maximum with 170-290 ppm of catalyst. This means that the CNT coverage can be completed with this optimum amount. Additional catalyst is therefore unnecessary because it will promote also side reactions in the system like polymer-polymer crosslinking.

Sonication also plays an important role in the process, because it separates agglomerates into single nanotubes and at the same time provides the necessary energy for the hydrosilylation reaction. As shown in Figure 5 the polymer coverage process of the nanotubes proceeds rapidly and is almost complete in about 10 minutes. Prolonged sonication times have instead a limited effect on the extractability.

\subsection{MWCNT-SMP10 suspension in THF, m-xylene and chloroform}

From the results obtained in cyclohexane we can extend the concept to the other organic solvents analyzed. In THF and m-xylene the polymer-solvent interaction is stronger than for polymer-CNT and SMP10 remains dispersed in solution without or only partially adhering on 
the nanotubes wall. The addition of $\operatorname{Pt}(0)$ is uninfluential and does not help to reach the needed "critical CNT coverage" that allows CNT suspension in the specific solvent.

In the case of chloroform a dark stable suspension can be obtained without $\operatorname{Pt}(0)$ addition as shown in Figure 6D. Different from the other organic solvents used, chloroform's density (1.49 $\left.\mathrm{g} / \mathrm{cm}^{3}\right)$ is almost double compared to cyclohexane $\left(0.779 \mathrm{~g} / \mathrm{cm}^{3}\right)$, THF $\left(0.89 \mathrm{~g} / \mathrm{cm}^{3}\right)$ or m-xylene $\left(0.86 \mathrm{~g} / \mathrm{cm}^{3}\right)$. Its value is also higher than the density of SMP10 $\left(0.998 \mathrm{~g} / \mathrm{cm}^{3}\right)$ and comparable with the density of MWCNT that can be ranged between $1.5-2.3 \mathrm{~g} / \mathrm{cm}^{3}$ [47]. This greatly affects the precipitation. This can be simply described by Archimedes' principle $F=g \cdot m \cdot\left(\rho_{P}-\rho_{F}\right) / \rho_{P}-$ $F_{D}$ stating that the force exerted on an object in a liquid is proportional to the difference between its density $\left(\rho_{\mathrm{P}}\right)$ and the density of the fluid $\left(\rho_{\mathrm{F}}\right)$ minus the drag $\left(\mathrm{F}_{\mathrm{D}}\right)$. Because partially covered $\rho_{P}$ is calculated as the weighted average between polymer and CNT density resulting in an almost negligible or negative precipitation of the nanotubes.

The suspension is anyway not homogeneous, suggesting that the polymer coverage is incomplete and aggregates can be found in the supernatant. A complete coverage and uniformly dispersed single nanotubes are instead obtained after the addition of $\operatorname{Pt}(0)$. The high extractability values obtained are also an effect of the chloroform density that suspends a higher nanotube amount up to $0.50 \mathrm{mg} / \mathrm{ml}$. Among these, a fraction with only partial coverage and/or still entangled is also most likely included but can not be easily separated from the rest via centrifugation.

\subsection{MWCNT-Ceraset suspensions}

The use of polysilazane Ceraset confirms the results obtained with polycarbosilane SMP10. With a similar chemical structure, stable and homogeneous suspensions of MWCNT in organic solvents can be easily achieved via sonication in the presence of $\operatorname{Pt}(0)$. The best result was obtained in THF where nanotubes without catalyst addition could be already separated and 
suspended. This indicates a higher non-covalent interaction of the polymer with the CNT instead than with the solvent. In this way the polymer can adhere onto the CNT wall and oppose the Van der Waals force via steric repulsion. The effectiveness in dispersing CNT via simple sonication in THF+Ceraset was already reported by Katsuda et al. [16] to produce SiCN-CNT ceramic composites but the dispersion process was not analyzed. In Table 1 it is shown that with the use of $\operatorname{Pt}(0)$ use the fraction of well dispersed CNT increases from $57 \%$ to $95 \%$ thanks to the induced hydrosilylation reaction.

In cyclohexane and chloroform a stable suspension was obtained only with $\operatorname{Pt}(0)$ addition indicating that it was fundamental to reach the necessary "critical surface coverage".

Similarly to SMP10, with Ceraset not only Baytubes but also Nanocyl were successfully suspended in cyclohexane. This suggests that the suspension method is generally valid and not specific for a specific type of CNT. Of course, this will lead to different extractabilities accordingly to the CNT characteristics such as purity, dimension and chirality.

\section{Conclusions}

We have demonstrated a simple straightforward strategy for the suspension of MWCNT in organic solvents with the use of preceramic polymers and $\operatorname{Pt}(0)$ catalyst. Nanotube suspension is directly achieved in about $10 \mathrm{~min}$ in one-step without the necessity of previous functionalization. The mechanism and the principal findings can be summarized as follow:

1) $\operatorname{Pt}(0)$ is fundamental to achieve a stable suspension in organic solvents. The use of SMP10 or Ceraset alone is not sufficient to efficiently suspend the CNT and better results were always achieved when using $\operatorname{Pt}(0)$.

2) The suspension mechanism is explained as the sum of a non-covalent assembly of the polymer on the CNT surface due to $\mathrm{CH}-\pi$ interaction, plus the hydrosilylation reaction between $\mathrm{Si}-\mathrm{H}$ group of the polymer and $\mathrm{C}=\mathrm{C}$ of the nanotube catalyzed by $\mathrm{Pt}(0)$. Non- 
covalent interaction were in fact demonstrated to not efficiently cover the nanotubes and assure sufficient steric repulsion. On the contrary with the addition of catalyst a higher and stable polymer coverage of the nanotubes can be achieved. When this is higher than a certain "critical coverage value" defined as the minimum polymer amount necessary on the CNT surface to avoid CNT aggregation, a stable suspension is obtained.

3) Using SMP10 the higher CNT concentrations were, respectively, $0.50 \mathrm{mg} / \mathrm{ml}$ in chloroform and $0.41 \mathrm{mg} / \mathrm{ml}$ cyclohexane. For Ceraset $0.47 \mathrm{mg} / \mathrm{ml}, 0.29 \mathrm{mg} / \mathrm{ml}$ and $0.26 \mathrm{mg} / \mathrm{ml}$ were obtained, respectively, in THF, cyclohexane and chloroform. In all the analyzed suspension the particle size distribution was $<1 \mu \mathrm{m}$.

4) This novel method is a simple and quick alternative to surfactant and CNT functionalization in organosilicon composites production. In addition it can be easily upscaled, all the materials are relatively cheap, commercially available and more importantly it can most likely be extended to polymer and molecules with the same functional groups.

\section{Outlook and possible applications}

The analyzed CNT-polymer suspensions process allows to have a stable and homogeneous distribution of filler in different organic solvents such as cyclohexane, chloroform and THF that can be used for different applications. As recently reported by Soraru et al. [48] and Zera et al. [49] highly porous aerogel are produced from preceramic polymers crosslinked with divinylbenzene (DVB) in suitable solvents. The cited method can be applied also to the CNT suspension to produce a novel MWCNT-polycarbosilane composite aerogel. The sample in Figure 7A has a filler content of $0.3 \%$ and was realized as proof of concept. The grayish uniform color instead of the characteristic white of SMP10-DVB aerogel suggests the effective incorporation of the filler. In the same picture is also visible half of the aerogel after pyrolysis 
in Argon atmosphere at $1000^{\circ} \mathrm{C}$. The shrinkage due to the gas released during the process is about $30 \%$ linear but the porous structure is maintained. Polymer derived ceramics (PDCs) carbon-rich aerogels are studied as suitable material for Li-ion batteries because as described by Pradeep et al. [50] the porous nature allows for fast ionic transport and helps to accommodate the structural changes which in turn allow a stable performance during repeated lithiation/delithiation.

Further applications of the dispersion can be found after the removal of the solvent. As shown in Figure 7 the obtained black liquid can be easily spread on different surfaces like glass and polystyrene or cast in a mould to realize a polymer-CNT composite with desired shape. The $\operatorname{Pt}(0)$ already included in the suspension process allows the mixture to cure at ambient temperature as described by Blum et al.[51]. The maximum achievable filler load was $1.4 \mathrm{wt}$. $\%$ and $1.3 \mathrm{wt} . \%$ for SMP10 in chloroform and Ceraset in THF, respectively. As comparison, 1.2 wt $\%$ is reported to be sufficient to reach percolation in PDMS[52]. Furthermore, the preceramic polymeric matrix can be converted into a SiC-CNT or SiCN-CNT ceramic matrix composite via pyrolysis[20]. Fields of interest are ceramic fibers [53], ceramic MEMS [54] and biomedical devices[55].

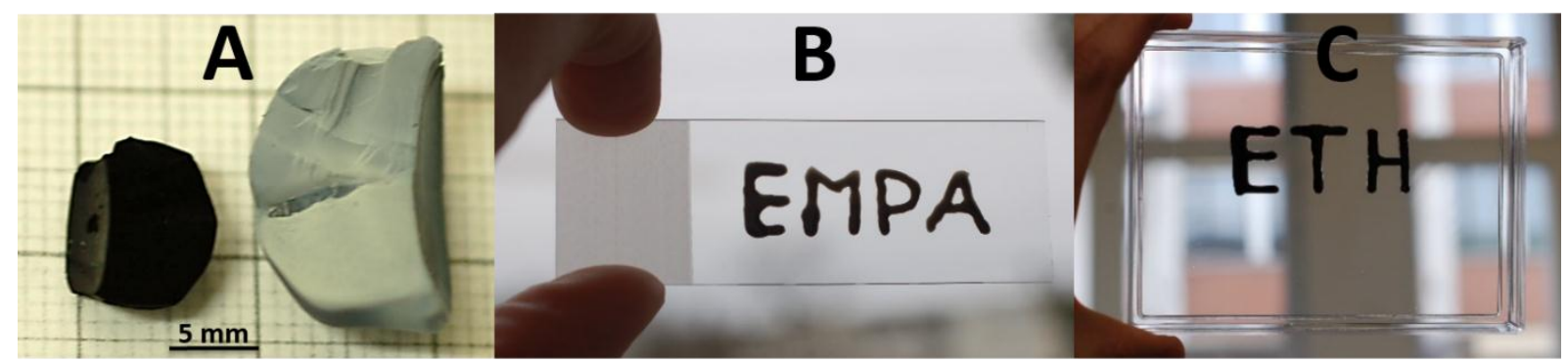

Figure 7: example of CNT suspension use. A) SMP10-DVB Aerogel with $0.3 \%$ CNT filler before and after $1000^{\circ} \mathrm{C}$ pyrolysis, respectively right and left. B) C) SMP10-CNT 1 wt\% filler content used as ink respectively on glass and polystyrene substrate 


\section{Acknowledgment}

This work was supported by Swiss National Science Foundation under the project $\mathrm{N}^{\circ}$ 205321_138055/1 named CeraMed - Implantable Ceramic MEMS Electrodes for Cardiac Pace Makers. The NMR hardware was partially granted by the Swiss National Science Foundation (SNFS, grant No. 150638). The authors gratefully thank Daniel Rentsch and Beatrice Fisher from the Functional Polymer Department (Empa) for the NMR spectra and GPC analysis and Felix Rechberger from the Laboratory for Multifunctional Materials (ETH Zurich) for the supercritical drying of the aerogels. 


\section{References}

[1] J.K.W. Sandler, J.E. Kirk, I.A. Kinloch, M.S.P. Shaffer, A.H. Windle, Ultra-low electrical percolation threshold in carbon-nanotube-epoxy composites, Polymer, 44 (2003) 5893-5899. http://dx.doi.org/10.1016/S0032-3861(03)00539-1

[2] J. Shen, W. Huang, L. Wu, Y. Hu, M. Ye, Thermo-physical properties of epoxy nanocomposites reinforced with amino-functionalized multi-walled carbon nanotubes, Composites Part A: Applied Science and Manufacturing, 38 (2007) 1331-1336. http://dx.doi.org/10.1016/j.compositesa.2006.10.012

[3] S.W. Kim, T. Kim, Y.S. Kim, H.S. Choi, H.J. Lim, S.J. Yang, C.R. Park, Surface modifications for the effective dispersion of carbon nanotubes in solvents and polymers, Carbon, 50 (2012) 3-33. http://dx.doi.org/10.1016/j.carbon.2011.08.011

[4] P. Bilalis, D. Katsigiannopoulos, A. Avgeropoulos, G. Sakellariou, Non-covalent functionalization of carbon nanotubes with polymers, RSC Advances, 4 (2014) 2911-2934. http://dx.doi.org/10.1039/C3RA44906H

[5] J. Chen, H. Liu, W.A. Weimer, M.D. Halls, D.H. Waldeck, G.C. Walker, Noncovalent Engineering of Carbon Nanotube Surfaces by Rigid, Functional Conjugated Polymers, J Am Chem Soc, 124 (2002) 90349035. http://dx.doi.org/10.1021/ja026104m

[6] D. Baskaran, J.W. Mays, M.S. Bratcher, Noncovalent and Nonspecific Molecular Interactions of Polymers with Multiwalled Carbon Nanotubes, Chem Mater, 17 (2005) 3389-3397. http://dx.doi.org/10.1021/cm047866e

[7] M.J. O'Connell, P. Boul, L.M. Ericson, C. Huffman, Y. Wang, E. Haroz, C. Kuper, J. Tour, K.D. Ausman, R.E. Smalley, Reversible water-solubilization of single-walled carbon nanotubes by polymer wrapping, Chemical Physics Letters, 342 (2001) 265-271. http://dx.doi.org/10.1016/S0009-2614(01)00490-0

[8] L. Xu, Z. Ye, Q. Cui, Z. Gu, Noncovalent Nonspecific Functionalization and Solubilization of MultiWalled Carbon Nanotubes at High Concentrations with a Hyperbranched Polyethylene, Macromolecular Chemistry and Physics, 210 (2009) 2194-2202. http://dx.doi.org/10.1002/macp.200900460

[9] A.-M. Caminade, J.-P. Majoral, Dendrimers and nanotubes: a fruitful association, Chemical Society Reviews, 39 (2010) 2034-2047. http://dx.doi.org/10.1039/B926408F

[10] L.-A. Liew, Y. Liu, R. Luo, T. Cross, L. An, V.M. Bright, M.L. Dunn, J.W. Daily, R. Raj, Fabrication of SiCN MEMS by photopolymerization of pre-ceramic polymer, Sensors and Actuators A: Physical, 95 (2002) 120-134. http://dx.doi.org/10.1016/S0924-4247(01)00723-3

[11] F. Dalcanale, J. Grossenbacher, G. Blugan, M.R. Gullo, A. Lauria, J. Brugger, H. Tevaearai, T. Graule, M. Niederberger, J. Kuebler, Influence of carbon enrichment on electrical conductivity and processing of polycarbosilane derived ceramic for MEMS applications, Journal of the European Ceramic Society, 34 (2014) 3559-3570. http://dx.doi.org/10.1016/j.jeurceramsoc.2014.06.002

[12] V. Liebau-Kunzmann, C. Fasel, R. Kolb, R. Riedel, Lithium containing silazanes as precursors for SiCN:Li ceramics-A potential material for electrochemical applications, Journal of the European Ceramic Society, 26 (2006) 3897-3901. http://dx.doi.org/10.1016/j.jeurceramsoc.2005.11.008

[13] Sporian Microsystems, http://www.sporian.com/harsh.html, last access 10 January 2016

[14] P. Baldus, M. Jansen, D. Sporn, Ceramic fibers for matrix composites in high-temperature engine applications, Science, 285 (1999) 699-703. http://dx.doi.org/10.1126/science.285.5428.699

[15] L. An, W. Xu, S. Rajagopalan, C. Wang, H. Wang, Y. Fan, L. Zhang, D. Jiang, J. Kapat, L. Chow, B. Guo, J. Liang, R. Vaidyanathan, Carbon-Nanotube-Reinforced Polymer-Derived Ceramic Composites, Advanced Materials, 16 (2004) 2036-2040. http://dx.doi.org/10.1002/adma.200306241

[16] Y. Katsuda, P. Gerstel, J. Narayanan, J. Bill, F. Aldinger, Reinforcement of precursor-derived Si-C-N ceramics with carbon nanotubes, Journal of the European Ceramic Society, 26 (2006) 3399-3405. http://dx.doi.org/10.1016/j.jeurceramsoc.2005.10.014 
[17] Y. Li, L.a. Fernandez-Recio, P. Gerstel, V. Srot, P.A.v. Aken, G. Kaiser, M. Burghard, J. Bill, Chemical Modification of Single-Walled Carbon Nanotubes for the Reinforcement of Precursor-Derived Ceramics, Chem. Mater., 20 (2008) 5593-5599. http://dx.doi.org/10.1021/cm801125k

[18] L. Fernandez, Y. Li, M. Burghard, Z. Burghard, P. Gerstel, J. Bill, F. Aldinger, Dispersion of Carbon Nanotubes in Polysilazanes for the Preparation of Reinforced Si-C-N Composites, Key Engineering Materials, 403 (2009) 231-234. http://www.scientific.net/KEM.403.231

[19] D. Shopova-Gospodinova, Z. Burghard, T. Dufaux, M. Burghard, J. Bill, Mechanical and electrical properties of polymer-derived Si-C-N ceramics reinforced by octadecylamine - Modified single-wall carbon nanotubes, Composites Science and Technology, 71 (2011) 931-937. http://dx.doi.org/10.1016/j.compscitech.2011.02.013

[20] F. Dalcanale, J. Grossenbacher, G. Blugan, M.R. Gullo, J. Brugger, H. Tevaearai, T. Graule, J. Kuebler, CNT and PDCs: A fruitful association? Study of a polycarbosilane-MWCNT composite, Journal of the European Ceramic Society, 35 (2015) 2215-2224. http://dx.doi.org/10.1016/j.jeurceramsoc.2015.02.016

[21] Z. Burghard, D. Schön, P. Garstel, J. Bill, F. Aldinger, Polymer-derived Si-C-N ceramics reinforced by single-wall carbon nanotubes, International Journal of Materials Research, 97 (2006) 1667-1672. http://dx.doi.org/10.3139/146.101399

[22] B. Krause, M. Mende, P. Pötschke, G. Petzold, Dispersability and particle size distribution of CNTs in an aqueous surfactant dispersion as a function of ultrasonic treatment time, Carbon, 48 (2010) 27462754. http://dx.doi.org/10.1016/j.carbon.2010.04.002

[23] S. Attal, R. Thiruvengadathan, O. Regev, Determination of the Concentration of Single-Walled Carbon Nanotubes in Aqueous Dispersions Using UV-Visible Absorption Spectroscopy, Anal Chem, 78 (2006) 8098-8104. http://dx.doi.org/10.1021/ac060990s

[24] J. Yu, N. Grossiord, C.E. Koning, J. Loos, Controlling the dispersion of multi-wall carbon nanotubes in aqueous surfactant solution, Carbon, 45 (2007) 618-623. http://dx.doi.org/10.1016/j.carbon.2006.10.010

[25] R. Rastogi, R. Kaushal, S.K. Tripathi, A.L. Sharma, I. Kaur, L.M. Bharadwaj, Comparative study of carbon nanotube dispersion using surfactants, J Colloid Interf Sci, 328 (2008) 421-428. http://dx.doi.org/10.1016/j.jcis.2008.09.015

[26] Q. Cheng, S. Debnath, E. Gregan, H.J. Byrne, Ultrasound-Assisted SWNTs Dispersion: Effects of Sonication Parameters and Solvent Properties, The Journal of Physical Chemistry C, 114 (2010) 88218827. http://dx.doi.org/10.1021/jp101431h

[27] K.G. Dassios, P. Alafogianni, S.K. Antiohos, C. Leptokaridis, N.-M. Barkoula, T.E. Matikas, Optimization of Sonication Parameters for Homogeneous Surfactant-Assisted Dispersion of Multiwalled Carbon Nanotubes in Aqueous Solutions, The Journal of Physical Chemistry C, 119 (2015) 7506-7516. http://dx.doi.org/10.1021/acs.jpcc.5b01349

[28] L. Dumée, K. Sears, J. Schütz, N. Finn, M. Duke, S. Gray, Influence of the Sonication Temperature on the Debundling Kinetics of Carbon Nanotubes in Propan-2-ol, Nanomaterials, 3 (2013) 70. http://dx.doi.org/10.3390/nano3010070

[29] I. Hiroshi, M. Fumikazu, T. Haruo, I. Toshikatsu, Oxidation Reaction of Polycarbosilane, Silicon-Based Polymer Science, American Chemical Society1989, pp. 619-637. http://dx.doi.org/10.1021/ba-19900224.ch034

[30] H. Wang, X.-d. Li, X.-x. Li, B. Zhu, D.-p. Kim, The kinetics of oxidation curing of polycarbosilane fibers, Korean Journal of Chemical Engineering, 21 (2004) 901-904. http://dx.doi.org/10.1007/BF02705537

[31] Y. Min, M. Akbulut, K. Kristiansen, Y. Golan, J. Israelachvili, The role of interparticle and external forces in nanoparticle assembly, Nat Mater, 7 (2008) 527-538. http://dx.doi.org/10.1038/nmat2206

[32] M. Nishio, M. Hirota, Y. Umezawa, The $\mathrm{CH} /[\mathrm{pi}]$ interaction : evidence, nature, and consequences, New York : Wiley1998.

[33] A. Beigbeder, M. Linares, M. Devalckenaere, P. Degée, M. Claes, D. Beljonne, R. Lazzaroni, P. Dubois, $\mathrm{CH}-\pi$ Interactions as the Driving Force for Silicone-Based Nanocomposites with Exceptional Properties, Advanced Materials, 20 (2008) 1003-1007. http://dx.doi.org/10.1002/adma.200701497 
[34] L.V. Interrante, K. Moraes, Q. Liu, N. Lu, A. Puerta, L.G. Sneddon, Silicon-based ceramics from polymer precursors, Pure and Applied Chemistry, 74 (2002) 2111-2117. http://dx.doi.org/10.1351/pac200274112111

[35] J.-T. Sun, C.-Y. Hong, C.-Y. Pan, Surface modification of carbon nanotubes with dendrimers or hyperbranched polymers, Polymer Chemistry, 2 (2011) 998-1007. http://dx.doi.org/10.1039/COPY00356E [36] B. Marciniec, H. Maciejewski, Hydrosilylation a comprehensive review on recent advances, Springer, Dordrecht, 2009. http://dx.doi.org/10.1007/978-1-4020-8172-9

[37] Y. Lee, K.-S. Jeon, H. Lim, H.S. Shin, S.M. Jin, H.R. Byon, Y.D. Suh, H.C. Choi, Silencing of Metallic Single-Walled Carbon Nanotubes via Spontaneous Hydrosilylation, Small, 5 (2009) 1398-1402. http://dx.doi.org/10.1002/smll.200801763

[38] S.R. Shah, R. Raj, Nanodevices that explore the synergies between PDCs and carbon nanotubes, Journal of the European Ceramic Society, 25 (2005) 243-249. http://dx.doi.org/10.1016/j.jeurceramsoc.2004.08.006

[39] J. Yin, S.-H. Lee, L. Feng, Y. Zhu, X. Liu, Z. Huang, S.-Y. Kim, I.-S. Han, The effects of SiC precursors on the microstructures and mechanical properties of $\mathrm{SiCf} / \mathrm{SiC}$ composites prepared via polymer impregnation and pyrolysis process, Ceramics International, 41 (2015) 4145-4153. http://dx.doi.org/10.1016/j.ceramint.2014.11.112

[40] R. Lee, Carbosilanes: Reactions \& Mechanisms of SMP-10 Pre-Ceramic Polymers, Marshall Space Flight Centre, 2009.

[41] G. Chollon, R. Pailler, R. Naslain, F. Laanani, M. Monthioux, P. Olry, Thermal stability of a PCS-derived SiC fibre with a low oxygen content (Hi-Nicalon), Journal of Materials Science, 32 (1997) 327-347. http://dx.doi.org/10.1023/A:1018541030308

[42] S. Kaur, R. Riedel, E. Ionescu, Pressureless fabrication of dense monolithic SiC ceramics from a polycarbosilane, Journal of the European Ceramic Society, 34 (2014) 3571-3578. http://dx.doi.org/10.1016/j.jeurceramsoc.2014.05.002

[43] H. Ly, R. Taylor, R.J. Day, F. Heatley, Conversion of polycarbosilane (PCS) to SiC-based ceramic Part II Pyrolysis and characterisation, Journal of Materials Science, 36 (2001) 4045-4057. http://dx.doi.org/10.1023/A:1017994810727

[44] M. Monthioux, O. Delverdier, Thermal behavior of (organosilicon) polymer-derived ceramics. V: Main facts and trends, Journal of the European Ceramic Society, 16 (1996) 721-737. http://dx.doi.org/10.1016/0955-2219(95)00186-7

[45] E. Bouillon, F. Langlais, R. Pailler, R. Naslain, F. Cruege, P.V. Huong, J.C. Sarthou, A. Delpuech, C. Laffon, P. Lagarde, M. Monthioux, A. Oberlin, Conversion Mechanisms of a Polycarbosilane Precursor into an Sic-Based Ceramic Material, Journal of Materials Science, 26 (1991) 1333-1345. http://dx.doi.org/10.1007/BF00544474

[46] L. He, Z. Zhang, X. Yang, L. Jiao, Y. Li, C. Xu, Liquid polycarbosilanes: synthesis and evaluation as precursors for SiC ceramic, Polymer International, 64 (2015) 979-985. http://dx.doi.org/10.1002/pi.4889

[47] C. Laurent, E. Flahaut, A. Peigney, The weight and density of carbon nanotubes versus the number of walls and diameter, Carbon, 48 (2010) 2994-2996. http://dx.doi.org/10.1016/j.carbon.2010.04.010

[48] G.D. Sorarù, F. Dalcanale, R. Campostrini, A. Gaston, Y. Blum, S. Carturan, P.R. Aravind, Novel polysiloxane and polycarbosilane aerogels via hydrosilylation of preceramic polymers, J. Mater. Chem., 22 (2012) 7676-7680. http://dx.doi.org/10.1039/C2JM00020B

[49] E. Zera, R. Campostrini, P.R. Aravind, Y. Blum, G.D. Sorarù, Novel SiC/C Aerogels Through Pyrolysis of Polycarbosilane Precursors, Advanced Engineering Materials, 16 (2014) 814-819. http://dx.doi.org/10.1002/adem.201400134

[50] V.S. Pradeep, D.G. Ayana, M. Graczyk-Zajac, G.D. Soraru, R. Riedel, High Rate Capability of SiOC Ceramic Aerogels with Tailored Porosity as Anode Materials for Li-ion Batteries, Electrochim Acta, 157 (2015) 41-45. http://dx.doi.org/10.1016/j.electacta.2015.01.088 
[51] Y.D. Blum, D.B. MacQueen, H.-J. Kleebe, Synthesis and characterization of carbon-enriched silicon oxycarbides, Journal of the European Ceramic Society, 25 (2005) 143-149. http://dx.doi.org/10.1016/j.jeurceramsoc.2004.07.019

[52] G.-X. Chen, H.-S. Kim, B.-H. Park, J.-S. Yoon, Highly insulating silicone composites with a high carbon nanotube content, Carbon, 44 (2006) 3373-3375. http://dx.doi.org/10.1016/j.carbon.2006.07.007

[53] H.-z. Wang, X.-d. Li, J. Ma, G.-y. Li, T.-j. Hu, Multi-walled carbon nanotube-reinforced silicon carbide fibers prepared by polymer-derived ceramic route, Composites Part A: Applied Science and Manufacturing, 43 (2012) 317-324. http://dx.doi.org/10.1016/j.compositesa.2011.12.007

[54] M. Schulz, Polymer derived ceramics in MEMS/NEMS - a review on production processes and application, Advances in Applied Ceramics: Structural, Functional \& Bioceramics, 108 (2009) 454-460. http://dx.doi.org/10.1179/174367609X422171

[55] J. Grossenbacher, M.R. Gullo, F. Dalcanale, G. Blugan, J. Kuebler, S. Lecaudé, H. Tevaearai Stahel, J. Brugger, Cytotoxicity evaluation of polymer-derived ceramics for pacemaker electrode applications, Journal of Biomedical Materials Research Part A, 103 (2015) 3625-3632. http://dx.doi.org/10.1002/jbm.a.35477 